\title{
PHARMACOGENOMICS: CURRENT ACTIONABLE VARIANTS
}

\author{
Vanessa González-Covarrubias ${ }^{1 *}$, Karla lozano ${ }^{1}$, Tomas Texis ${ }^{1}$, Cintia K. Guzmán-Cruz ${ }^{1}$, \\ Mauricio Rodríguez-Dorantes ${ }^{1}$, KenNeth Rubio-Carrasco ${ }^{2}$, Luz H. MÉndez-Lorenzo ${ }^{1}$, \\ AND XAVIER SOBERóN ${ }^{1}$ \\ ${ }^{1}$ Instituto Nacional de Medicina Genómica, SSA, Mexico City; ${ }^{2}$ Department of Pharmacy, Faculty of Chemistry, \\ Universidad Nacional Autónoma de México (UNAM), Mexico City, Mexico
}

\begin{abstract}
Pharmacogenomics (PGx), one of the several tools of precision medicine, has been slowly implemented in the clinic during the past decades. This process generally starts with direct and indirect genotype-phenotype associations of gene variants and drug efficacy, or adverse drug reactions, followed by replication and validation studies. Institutional efforts led by the PGx Research Network, The PGx Knowledge Base, and The Clinical Pharmacogenetics Implementation Consortium, mine all available data for further validation or research in additional populations. This data mining gives rise to a detailed classification of over 200 druggene pairs which, with enough documentation, may become part of a publishable guideline to aid clinicians in drug selection and dosing using genetics. The US Food and Drug Administration utilizes these guidelines to issue warnings and recommendations for specific drugs and their cautioning serves clinicians and pharmacists worldwide. Here, we aim to discuss the steps of this process and list existing actionable drug-gene pairs. Moreover, we describe the current status of PGx knowledge in populations from Mexico for actionable variants on the 19 genes listed by present PGx guidelines affecting 47 drugs. Our review collects current allele frequency information for these actionable variants, lists gaps of PGx information for relevant markers, and highlights the importance of continuing PGx research in Native and Mestizo populations. (REV INVEST CLIN. 2020;72(5):271-9)
\end{abstract}

Key words: Pharmacogenomics. Drug efficacy. Pharmacokinetics. Pharmacodynamics. Mestizos. Natives.

\section{INTRODUCTION}

Pharmacogenetics, a term coined in 1957 by Friedrich Vogel $^{1}$, refers to the study of the genetics of variable drug response and is used interchangeably with pharmacogenomics (PGX), though the latter has a broader scope considering the impact of multiple variants across the genome. In this review, we will use the term PGx to refer to either concept ${ }^{2}$.
Initial observations on the relationship between drug efficacy and metabolic individuality dates back to the work of Motulsky and Garrod at the end of the $19^{\text {th }}$ century ${ }^{3}$. The broader use of the terms pharmacogenetics in 1957 and PGx in $1997^{4}$ supported the formality and the development of this discipline. Several decades of research in the field have led to the identification, characterization, validation, and implementation of dozens of genetic biomarkers to improve
*Corresponding author:

Vanessa González-Covarrubias

E-mail: vgonzalez@inmegen.gob.mx
Received for publication: 4-09-2019

Approved for publication: 9-01-2020

DOI: $10.24875 / R I C .20003239$

0034-8376 / (c) 2020 Revista de Investigación Clínica. Published by Permanyer. This is an open access article under the CC BY-NC-ND license (http://creativecommons.org/licenses/by-nc-nd/4.0/). 
Figure 1. Growth of pharmacogenomics (PGx) published research overtime. Proportion of publications in PGx compared to those in cancer. The rate of growth of PGx reports overtime parallels that of cancer research. CPIC: Clinical Pharmacogenetics Implementation Consortium.

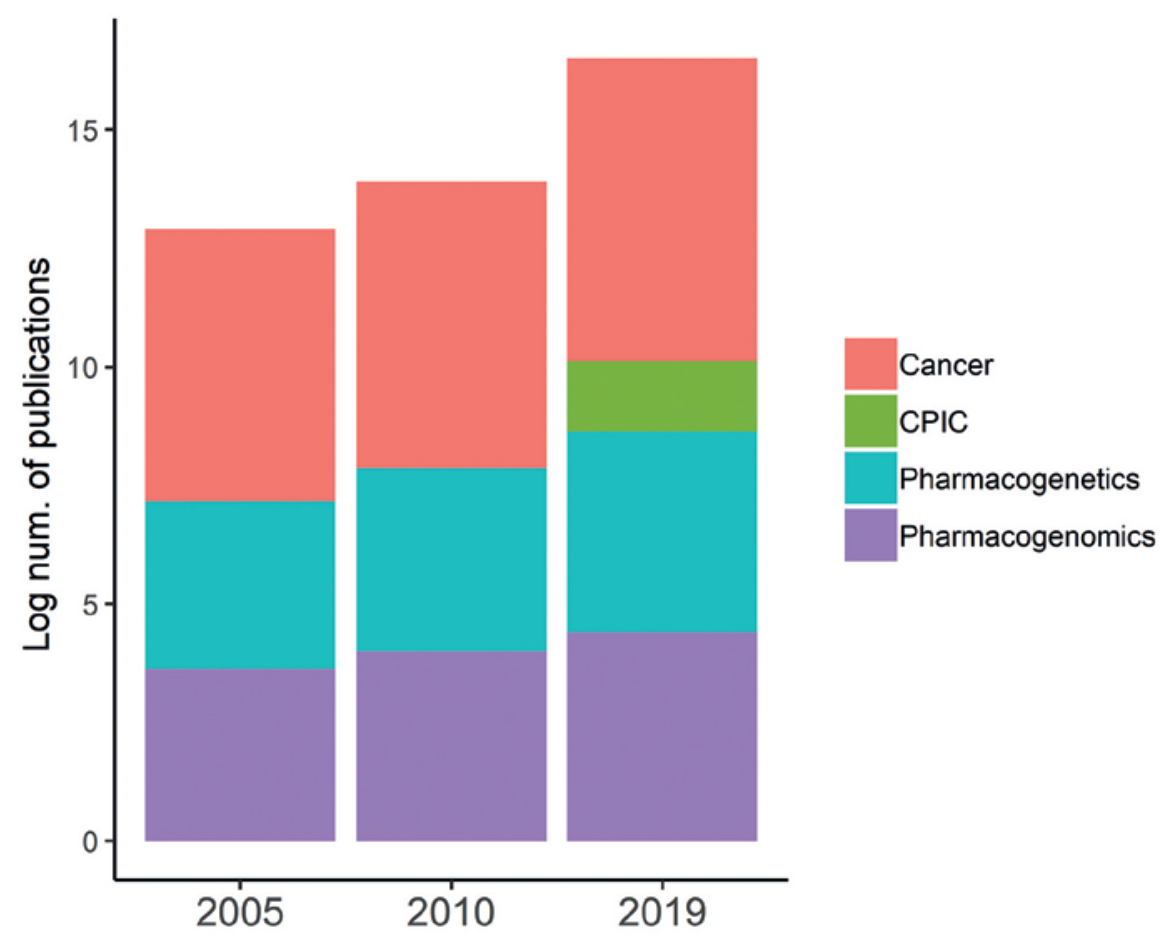

drug selection and efficacy and to prevent adverse drug reactions (ADRs). Up to June 2019, the scientific literature listed over 5500 articles on PGx, of which almost half were published in the past 5 years (Fig. 1). Recent studies cover different populations, although most efforts have focused on populations where technologies were more prevalent.

The advent of genomic technologies has facilitated the investigation of genes influencing drug safety and efficacy and its liaison with phenotyping assays. At present, it is possible to use pharmacogenetic information to personalize drug prescription for over 40 drugs using a varied arrange of genomic platforms, such as probe genotyping, microarrays, Sanger sequencing, and next-generation sequencing. These markers include single-nucleotide polymorphisms, copy-number variations, short insertions or deletions, and variable number tandem repeats, affecting a drug's pharmacodynamics (PD) or pharmacokinetics (PK). Genes affecting PK usually refer to enzymes involved in the absorption, distribution, metabolism, or elimination and include transporters and members of the CYP450 family (Table 1 ). Genes that affect a drug's target (PD) are intrinsic to the drug and can include enzymes, proteins, or receptors. For example, tamoxifen, used to treat breast cancer, binds to the estrogen receptor (ESR1) to prevent its activation. Furthermore, tamoxifen is a prodrug activated by CYP2D6; thus, CYP2D6 and ESR1 are part of tamoxifen pharmacogenetics (Table 1). Compared to PD, PK has been more intensely characterized so that it is possible to stratify patients into four phenotypes, poor, intermediate, extensive, or ultra-rapid phenotypes (Fig. 2) ${ }^{5}$.

As technology advances and results of the $1000 \mathrm{Ge}-$ nomes Project become available, research points toward the simultaneous use of hundreds of markers for tailoring diagnosis and treatment. Here, we will review actionable PGx markers, their classification, the process involved in the making of an actionable marker, institutions involved, and examples. Finally, we will complement this list with current knowledge of allele frequencies for Mexican populations and its potential impact for dosing or prescription. 
Figure 2. Metabolism capacity (phenotypes) population distribution. Population distribution of variable metabolic activities (absorption, distribution, metabolism, or elimination). These differences give rise to four major genotypes.

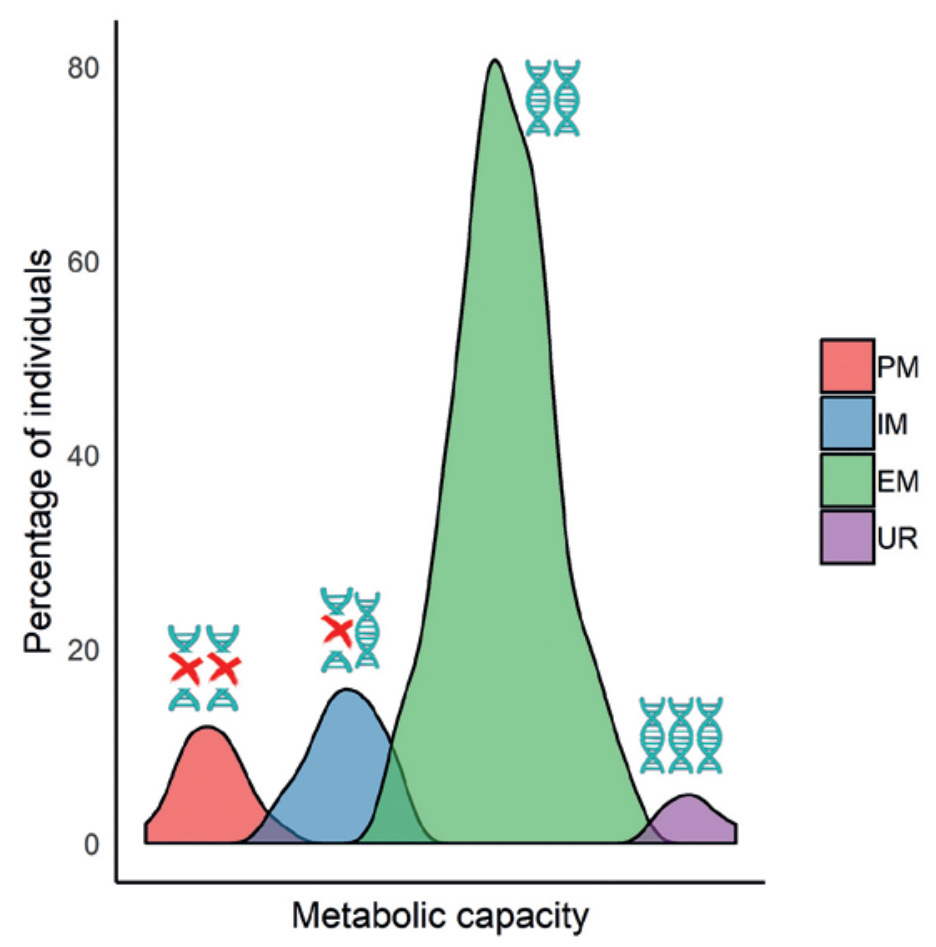

\section{DRUG-GENE PAIRS AND THE FOOD AND DRUG ADMINISTRATION (FDA)}

Most pharmacogenetic markers are directly or indirectly involved in the PK, PD, or ADRs of medications connecting a gene and its variants with a pharmacological outcome, generating a drug-gene pair. For example, typical drug-gene pairs include warfarin, a widely used anticoagulant, and gene variants $C Y$ P2C9*2, CYP2C9*3, CYP2C9*5, and VKORC1*2-4 represent part of the main picture of warfarin's pharmacokinetic and pharmacodynamic pathways. These, together with demographic characteristics, are capable of defining a tailored dose using PGx algorithms (e.g., warfarindosing.org) ${ }^{6}$. An example of a druggene pair related to ADRs is abacavir and $H L A-B * 5701$. Patients with this HLA allele are strongly warned against the use of abacavir as they will likely present a lethal hypersensitivity reaction ${ }^{7}$ (Tables 1 and 2).

The USA FDA, equivalent to most world drug regulatory agencies, has become an advocate for the use of PGx by including genetic information on drug labels promoting the understanding of how these markers contribute to drug responses ${ }^{8,9}$. Since 2014, the FDA has been increasingly approving "personalized medicines," i.e., drugs for which PGx testing is necessary before prescription ${ }^{10}$. At present, there are 261 druggene pairs listed on the FDA website with pharmacogenetic information ${ }^{11}$ (/www.fda.gov/media/107901/ download), but only a few require testing and have an official implementation guideline (Table 2 ). To be implemented, a clinically useful drug-gene pair requires a strong genotype-phenotype association followed by ample replication studies and validation.

To prioritize research and implementation policies, the classification of pharmacogenetic markers has been prompted by several researchers. For example, Haga and Burke classified PGx tests according to their biological foundation as acquired or inherited variants, alone or in combination, preemptive testing, and incidental or ancillary information ${ }^{13}$, but this classification did not stick to the current trends. Moreover, in its own classification effort, the FDA classifies PGx testing in (i) required, when genetic testing or functional 
Table 1. Most studied PGx genes classified by their main drug pathway

\begin{tabular}{|c|c|c|c|c|c|}
\hline \multicolumn{3}{|c|}{ Pharmacokinetics } & \multicolumn{3}{|c|}{ Pharmacodynamics/ADRs } \\
\hline DPYD & CES2 & SLC47A1 & $R Y R 1$ & GRK4 & DRD2 \\
\hline$A B C A 1$ & TPMT & CYP2C9 & $E G F R$ & VDR & NPR1 \\
\hline COMT & CYP3A4 & NAT2 & ESR 1 & $A C E$ & $D R D 1$ \\
\hline$A B C G 1$ & CYP2B6 & $A B C G 2$ & RYR2 & $N R 3 C 2$ & PTGIS \\
\hline UGT $1 A 1$ & POR & CYP1A2 & $D B H$ & HSD 11B2 & APOA 1 \\
\hline CYP2D6 & $S L C 22 A 1$ & G6PD & PEAR1 & CRHR1 & $A D R B 2$ \\
\hline$A B C B 1$ & CYP3A5 & MAOA & CACNA1S & ARID5B & VKORC1 \\
\hline
\end{tabular}

PGx: pharmacogenomics; ADRs: adverse drug reactions.

protein assays ought be conducted before using the drug (e.g., trastuzumab - HER2 and clopidogrel CYP2C19); (ii) recommended testing, highly useful to define dosing or preventing ADRs (e.g., azathioprine - TPMT and citalopram - CYP2D6); (iii) actionable, PGx information on dosing or toxicity due to genetic variants but does not mention genetic testing; and (iv) informative, mentions a gene or protein associated to the PK/PD of a drug but without variantspecific information. These tests are not FDA approved, but are regulated by the Clinical Laboratory Improvement Amendment of $1988^{14}$. Of the 261 drug-gene pairs, $35 \%$ are for oncology, followed in proportion by antipsychotic, cardiovascular, metabolic, gastroenterology, hematology, urology, and autoimmune diseases. The most common PGx markers other than those for cancer treatment are located on genes CYP2D6, CYP2C19, CYP2C9, and UGT1A1, influencing at least 20 different drugs.

Since genotyping and interpretation of results for 261 drug-gene pairs might not be straightforward, research groups have tried to consolidate shorter variant lists to facilitate PGx implementation. One of these attempts is part of the electronic Medical Records and Genomics network, which selected 82 pharmacogenes for preemptive sequencing in 5000 subjects. A selected list of these 82 genes is shown in Table 1 and represents a major part of the core of PG $x^{15}$.

\section{PHARMACOGENETIC GUIDELINES}

The PGx knowledge base (PGKB) is an organization created over 18 years ago and is one of the largest resources of PGx data. Its website (www.pharmgkb.org) publishes PGx information, including drug-gene pairs, phenotypes, pathways, dosing guidelines, drug labels, and variant and clinical annotations, among other data. Note that, the concept of "actionable marker" is distinctively addressed by the FDA and the PGKB; the former usually refers to testing, while the PGKB refers to its clinical utility or actionability in practice ${ }^{16}$.

PGx markers listed by FDA come from two major platforms developed by the PGx Research Network which has under its wing, the PGKB, and the Clinical Pharmacogenetics Implementation Consortium (CPIC), the latter formed in $2009^{17}$. The role of the PGKB is to collect, mine, annotate, curate, and assign a validation level to genetic markers of drug response according to the amount of clinical evidence associated to a drug trait. Their website currently lists genetic information for 309 drugs, although only markers with the highest level of validation have been medically endorsed and are included in implementation guidelines written and published by the CPIC ${ }^{18,19}$.

One step further to the advancement of PGx implementation is approached by the CPIC from which the FDA takes genetic information for drug labeling, warnings, and testing recommendations. Since CPICs goal is to develop and implement dosing guidelines, they also classify drug pairs according to their potential inclusion in a clinical setting as; "A" variants, with high evidence that should be used to change drug prescription; "B" variant, that could be used to improve prescription due to the availability of therapeutic alternatives; "C" variant, with some evidence but not yet convincing, impractical without clear drug alternatives; and "D" drug-gene variants, with few studies reported thus, unclear clinical actions. In brief, 
Table 2. Current drug-gene pairs with CPIC guidelines for clinical implementation

\begin{tabular}{|c|c|c|}
\hline Year/Update & Gene & Drug(s) \\
\hline $2011 / 2017$ & CYP2C9, VKORC1, CYP4F2 & Warfarin \\
\hline $2013 / 2017$ & $D P Y D$ & Fluoropyrimidines \\
\hline $2013 / 2017$ & $H L A-A, H L A-B$ & Carbamazepine, oxo-carbamazepine \\
\hline 2013 & CYP2C19 & Clopidogrel \\
\hline 2013 & IFNL3 & Peginterferon-alpha-based regimens \\
\hline $2013 / 2018$ & TPMT, NUDT15 & Thiopurines \\
\hline 2014 & CYP2C9, HLA-B & Phenytoin \\
\hline 2014 & CYP2D6 & Codeine \\
\hline 2014 & G6PD & Rasburicase \\
\hline $2014 / 2017$ & CFTR & Ivacaftor \\
\hline 2014 & $H L A-B$ & Abacavir \\
\hline 2014 & SLCO1B1 & Simvastatin \\
\hline 2015 & CYP2D6, CYP2C19 & Selective serotonin reuptake inhibitors \\
\hline 2015 & CYP3A5 & Tacrolimus \\
\hline 2015 & $H L A-B$ & Allopurinol \\
\hline 2015 & UGT1A1 & Atazanavir \\
\hline 2016 & CYP2C19 & Voriconazole \\
\hline 2016 & CYP2D6 & Ondansetron \\
\hline 2016 & CYP2D6, CYP2C19 & Tricyclic antidepressants \\
\hline 2018 & CYP2D6 & Tamoxifen \\
\hline 2018 & RYR1, CACNAC1S & Volatile anesthetic agents \\
\hline 2019 & CYP2B6 & Efavirenz \\
\hline 2019 & CYP2D6 & Atomoxetine \\
\hline
\end{tabular}

Adapted from https://cpicpgx.org/guidelines/.

CPIC guidelines aim to guide patient care decisions for specific drugs utilizing genetic information ${ }^{20}$. At present, there are 23 published CPIC guidelines for 47 drugs, which include 19 genes also classified as "VIP genes" (Tables 2 and 3 ).

\section{EXAMPLES OF ACTIONABLE PGX MARKERS}

The implementation of VKORC1, CYP4F2, and CYP2C9 markers for warfarin dosing was established in 2011 but has been rather controversial. Ample research has proven the strong association of variants on these genes and anticoagulant response or ADRs. Nevertheless, opposing results on the utility of PGX testing for warfarin by the USA ${ }^{21}$ and European ${ }^{22}$ studies were reported in 2013. Afterward, the CPIC published an update to this guideline suggesting the inclusion of continental ancestry for dose assessment ${ }^{23}$ and aiming for its implementation despite unsettling benefits.

Another interesting drug-gene pair is tamoxifen CYP2D6, for which a CPIC guideline was just published less than a year ago. Literature searches between 1982 and 2019 show almost 300 studies associating tamoxifen breast cancer response to genetic variation. Forty years of challenging research finally delivered a PGx guideline for clinical implementation. The barriers were many, including that tamoxifen is metabolized by dozens of enzymes but relies mostly on CYP2D6 to become activated. CYP2D6 is highly polymorphic with over 100 variants whose combinations can give rise to several metabolic phenotypes (Fig. 2) ${ }^{24}$. For example, individuals with the CYP2D6*9, *10, *14B, 
Table 3. Allele frequencies for selected actionable PGx markers in major populations

\begin{tabular}{|c|c|c|c|c|c|c|}
\hline Gene variant & $\begin{array}{c}\text { MAF } \\
\text { (Natives) }\end{array}$ & $\begin{array}{c}\text { MAF } \\
\text { (Mestizo) }\end{array}$ & $\begin{array}{c}\text { MAF } \\
(M X L / A M R)\end{array}$ & $\begin{array}{l}\text { MAF } \\
\text { (EUR) }\end{array}$ & $\begin{array}{l}\text { MAF } \\
\text { (YRI) }\end{array}$ & $\begin{array}{l}\text { MAF } \\
\text { (EAS) }\end{array}$ \\
\hline $\begin{array}{l}\text { CYP2C19*2 } \\
\text { rs4244285 }\end{array}$ & $0-0.310$ & 0 & 0.125 & 0.146 & 0.145 & 0.290 \\
\hline $\begin{array}{l}\text { CYP2C19*3 } \\
\text { rs4986893 }\end{array}$ & 0 & 0 & 0.003 & 0.006 & 0.007 & 0.083 \\
\hline $\begin{array}{l}\text { CYP2D6*4 } \\
\text { rs3892097 }\end{array}$ & $0.003-0.088$ & 0.011 & 0.107 & 0.181 & 0.033 & 0.648 \\
\hline $\begin{array}{l}\text { CYP2D } 6 * 5 \\
\text { gene deletion }\end{array}$ & $0-0.036$ & 0.020 & 0.021 & 0.028 & 0.062 & 0.051 \\
\hline $\begin{array}{l}\text { CYP } 3 A 5^{*} 3 \\
\text { rs776746 }\end{array}$ & NF & 0.730 & 0.800 & 0.920 & 0.150 & 0.710 \\
\hline $\begin{array}{l}T P M T * 3 A \\
\text { rs1800460 } \\
\text { rs1142345 }\end{array}$ & NF & $0.029-0.057$ & 0.0384 & 0.0331 & 0.0045 & 0.0003 \\
\hline $\begin{array}{r}\text { UGT1A1*28 } \\
\text { rs8175347 }\end{array}$ & NF & $0.334-0.360$ & 0.400 & 0.316 & 0.391 & 0.148 \\
\hline $\begin{array}{l}\text { VKORC1 } \\
\text { rs9923231 }\end{array}$ & $0.008-0.020$ & $0.050-0.190$ & 0.460 & 0.410 & 0.100 & 0.880 \\
\hline
\end{tabular}

A comprehensive list of all CPIC variants and its allele frequency in Mexican populations is presented in Supplementary Table 1 . MAF: minor allele frequency, NF: not found, "0" refers to an actual zero, MAF information comes from CPIC guidelines, the PGKB, HapMap 1000G or Genome Aggregation (gnomAD) databases, a full list with all level1 variants is found in Table S1. References to publications for each variant are also listed in Table S1.

PGx: pharmacogenomics; MLA: Mexicans from Los Angeles.

$* 17, * 29$, and $* 41$ alleles will have decreased activity; individuals with the CYP2D $*^{*} 3-* 8, * 11-* 13, * 15$, $* 19-* 21, * 36, * 38, * 40$, and $* 42$ alleles will have no activity, and all of the above will elicit a lower activation of tamoxifen and decreased efficacy. CYP2D6 alleles have large differences between populations in addition to full deletions, duplications, and the presence of the pseudogene CYP2D7, which complicates assigning its functional impact ${ }^{25}$. At present, specific CYP2D6 genotype combinations are classified and ranked for different "Activity Scores" (AS $=0.5-\geq 3$ ) which are translated into a course of action for clinical application.

Clinical PGx guideline implementation has been slightly straightforward for several drug pairs. For instance, ivacaftor, prescribed for cystic fibrosis, is a targeted agent, approved only in cases showing specific genetic markers, including cystic fibrosis transmembrane conductance regulator (CFTR) G551D26. Therefore, ivacaftor prescription requires genetic testing. Nevertheless, new and rare variants on CFTR are continuously being reported, and a 2017 update for this guideline included about a dozen of additional CFTR polymorphisms to complement the PGx testing for ivacaftor. Another example is the activation of clopidogrel, which takes place by at least six enzymes. Hepatic CYP2C19 activity is mostly responsible for this activation, hence, poor CYP2C19 metabolizers are at high risk of cardiovascular events due to a lack of clopidogrel activity and should be given an alternative drug. CYP2C19 has over 30 reported alleles, and available genetic testing can identify the most common alleles, ${ }^{*} 2, * 3, * 4 A / B, * 5-* 8$, and $* 17$, to provide with PGx guidance ${ }^{27}$. These examples are paralleled by many others ${ }^{24}$, supporting the prescription of personalized drugs with the aid of a PGx data network.

\section{ETHNIC DIFFERENCES IN PGX}

Variations in drug response between different populations have long been acknowledged. For example, $C Y$ $P 3 A 5^{*} 3$, affecting tacrolimus disposition, is 2 times more frequent in Asian populations compared to Europeans, and variant CYP2D6*10, impacting at least $25 \%$ 
of all prescription drugs, is more common among individuals from Malaysia and China compared to other continental groups. Caucasian populations require a $30 \%$ lower dose of warfarin compared to Africans due to CYP2C9 and VKORC1 polymorphisms ${ }^{28-30}$. These variants account for $35 \%$ dose variations in Europeans, $\sim 30 \%$ in Mexicans ${ }^{31}$, and $10 \%$ in African-Americans; . For the latter two, variants on NQO1, CALU, and GGCX may complement dose variation, highlighting the fact that certain genes distinctively impact on coumarin dosing according to population stratification. PGx research in Latin America has identified either similar frequencies on actionable markers among populations or striking differences. In this regard, variants on CYP3A5, VKORC1 rs9923231, CYP2C9, and SLCO1B1 show significant population differentiation (Fst) in admixed populations within Latin America compared to Europeans, Asians, or Africans ${ }^{29,30,32}$. Nevertheless, PGx differences are often 10- to 40-fold within individuals in any population, while differences between two ethnic groups are rarely $>2$ - or 3 -fold ${ }^{33}$. Hence, interindividual variability is much greater than group variation, a fact framing $P G x$ and all other complex traits so that an individualized treatment will consider interindividual variations above population differences ${ }^{29}$.

The notion that PGx would mostly benefit the outliers might have slowed down its implementation. Pharmacogenetic reports tend to list frequencies and statistics that may not necessarily highlight the relevance of a marker. For example, TPMT deficiency is a cause of severe ADRs in patients receiving thiopurines, which occurs in 1 of every 300 patients and the administration of anesthetics elicit malignant hyperthermia in 1 of every 2000 people due to variation in CACNA1S and $R Y R 1^{34}$. These statistics convey the idea of a low occurrence of PGx relevance. In this regard, the research groups of Ratain et al. and Roden et al. independently investigated actionable genotypes in over 10,000 patients and identified one or more actionable variants in $>90 \%$ of all individuals genotyped, emphasizing the benefits of "the outlier in all of us" and thus preemptive pharmacogenetic testing ${ }^{34.35}$.

\section{STATUS OF ACTIONABLE PGX MARKERS IN MEXICANS}

Population stratification for PGx relevant variants within Latin America has also been widely presented, for example, CYP3A5*3 and VKORC1 rs992323129, highlighting the identification and characterization of genetic variants that influence health, disease, and drug treatment as of paramount relevance for every single population ${ }^{37}$. In Mexico, Mexican Mestizos and Natives represent on average $80 \%$ and $10-15 \%$ of the population. Natives are categorized into 68 ethnic groups with low genetic diversity, but wide differentiation ${ }^{38}$. Novel variants, possibly private to Mexicans, have been described for CYP2C9, CYP2D6, and VKORC1 $1^{31,39-41}$ and could affect individual pharmacokinetic profiles and drug response. Nevertheless, Mexico does not have a program for PGx implementation. Recent updates to the federal or local health care laws (NOM 220-SSA, and the Hospital Pharmacy Program at ISSEMYM), mention PGx as a necessary tool for precision medicine, but no official policies have been issued from these initiatives, nor the adoption of those already published. Moreover, the identification of novel variants in individuals of Mexican descent is expected to increase as more genome and exome sequencing projects are completed. Today, we have an idea of this diversity given by the Exome Aggregation Consortium that reported over 600,000 genetic variants only in Mexican and Latin American individuals ${ }^{20}$. We sought to identify allele frequencies of the 144 variants in 19 genes of current CPIC guidelines in Mestizos other than MXL (Mexicans from Los Angeles) and in Natives. Table 3 summarizes allele frequencies of selected variants. A full list with all 144 variants is presented in Supplemental Table 1.

Interestingly, $12.5 \%$ (18) of all CPIC listed variants were not found in any population from Mexico. These were on CFTR and RYR1, 144 PGx markers have been listed for these genes, but no allele frequency (AF) information was found for Natives or Mestizos. (Table 3 and Supplemental Table 1).

Table 3 and Supplemental Table S1 summarize information on a higher AF of variants on VKORC1, CYP2D6*4, CYP2C9*3, or SLCO1B1*5 in Caucasians versus Mestizos or Natives, in contrast to higher $A F$ in Natives/Mestizos versus Caucasians, for CYP3A5 ${ }^{*} 1,{ }^{*} 7$, CYP2B6*4, HLA-B*1502, and HLA-B*5801. Moreover, variants CYP2C19*2/*3, CFTR G178R, S1251N, and TPMT*4 seem to be absent in Mexicans (Table 3 and Table S1). Similarities in AF have been reported for CYP $2 B 6^{*} 1 /{ }^{*} 6, \quad$ CYP $3 A 5^{*} 3 / 6, \quad$ TPMT $^{*} 1 /{ }^{*} 3 A$, and 
Table 4. Example of MAF differences in Natives (Zapotecos) and Mestizos

\begin{tabular}{|c|c|c|}
\hline PGx marker & MAF Zapotecos* & MAF Mestizos \\
\hline$H L A-A * 31: 01$ & 0.067 & $0.024-0.083(5)$ \\
\hline$H L A-B * 57: 01$ & 0 & $0-0.015(6)$ \\
\hline$H L A-B^{*} 15: 02$ & 0 & $0-0.009(7)$ \\
\hline$H L A-B * 58: 01$ & 0.022 & $0-0.100(5)$ \\
\hline CYP2C9*2 rs1799853 & 0 & 0.130 \\
\hline CYP2C9*3 rs1057910 & 0.012 & 0.130 \\
\hline SLCO1B1*5 rs4149056 & 0.044 & $0.109-0.153$ \\
\hline UGT1A1*80 rs887829 & 0.367 & 0.346 \\
\hline TPMT*2 rs1800462 $^{*}$ & 0 & $0-0.0375$ \\
\hline TPMT*3B rs 1800460 & 0.056 & $0.003-0.041$ \\
\hline TPMT*3C rs1142345 & 0.056 & $0.006-0.045$ \\
\hline TPMT*4 rs1800584 & 0 & 0 \\
\hline
\end{tabular}

*The Zapotecos' population size is close to 1 million. Many migration waves have pinpointed significant Zapoteco dwellings in several cities in California, USA.

PGx: pharmacogenomics.

UGT1A1*28, assuming similar PK/PD and ADRs as those observed for other populations. Nevertheless, these comparisons must be taken with caution as many determinations in Mexicans have been performed with a small sample size. Strikingly, fewer than $25 \%$ of the 144 PGx implemented variants were available for one or several native populations (Table S1). A scarce availability on AF data hinders comparisons between groups or populations, but prompts to direct endeavors to intensify PGx data collection in these individuals. As an example, we present AF in variants retrieved for Zapotecos, Native dwellers of the South and Center South of the country, to pinpoint similarities and differences when compared to Mestizos. Apparent differences were observed on HLA-B5801, CYP2C9*3, and SLCO1B1*5 (Table 4). It is expected that such comparisons are due to change as more research gathers information from natives.

\section{CONCLUSIONS}

PGx plays a major role in precision medicine. Shortterm endeavors may seek to complete a catalog of PGx variation worldwide to aid clinicians in the assignment of treatments with a higher success rate, according to the patient's genetic makeup. More likely, long-term efforts will dissect the whole human genome individually in a pre-emptive manner, in addition to the consideration of relevant factors such as the environment and the microbiome ${ }^{42}$.

The implementation of PGx in Latin America's admixed populations faces common difficulties, including access to technology, trained personnel, marker interpretation, and its inclusion in daily practice. Local implementation is also hindered by population stratification and a lack of complete registries of inhabitants' PGx variations and health-care records. A dweller of Mexico can show a Native/Caucasian ancestral component from 0.01 to 0.99 hampering the use of ancestry as a PGx proxy. Moreover, understudied populations may hold private variants not previously reported nor assessed for functional impact.

We envision the use of actionable variants listed by international curating institutions such as the PGKB and the CPIC, complemented with private and rare variants using sequencing strategies on local populations.

\section{ACKNOWLEDGMENTS}

This work was supported by CONACYT grants FOSSIS No.272795 and Ciencia Básica No. 252952. 


\section{SUPPLEMENTARY DATA}

Supplementary data are available at Revista de Investigación Clínica online (www.clinicalandtranslationalinvestigation.com). These data are provided by the corresponding author and published online for the benefit of the reader. The contents of supplementary data are the sole responsibility of the authors.

\section{REFERENCES}

1. Campion DP, Dowell FJ. Translating pharmacogenetics and pharmacogenomics to the clinic: progress in human and veterinary medicine. Front Vet Sci. 2019;6:22.

2. Pirmohamed M. Pharmacogenetics and pharmacogenomics. $\mathrm{Br}$ J Clin Pharmacol. 2001;52:345-7.

3. Piro A, Tagarelli G, Lagonia P, Quattrone A, Tagarelli A. Archibald Edward Garrod and alcaptonuria: "inborn errors of metabolism" revisited. Genet Med. 2010;12:475-6.

4. Hedgecoe AM. Terminology and the construction of scientific disciplines: the case of pharmacogenomics. Sci Technol Hum Values. 2003;33:34-9

5. Ehret M. The basics of pharmacogenomics: review the basics of genomics and available tests. Ment Heal Clin. 2012;1:207-9.

6. Finkelman BS, Gage BF, Johnson JA, Brensinger CM, Kimmel SE. Genetic warfarin dosing: tables versus algorithms. J Am Coll Cardiol. 2011:57:612-8

7. Martin MA, Klein TE, Dong BJ, Pirmohamed M, Haas DW, Kroetz $\mathrm{DL}$, et al. Clinical pharmacogenetics implementation consortium guidelines for HLA-B genotype and abacavir dosing. Clin Pharmacol Ther. 2012;91:734-8.

8. Evans WE, McLeod HL. Pharmacogenomics drug disposition, drug targets, and side effects. N Engl J Med. 2003;348:538-49.

9. Roden DM, Wilke RA, Kroemer HK, Stein CM. Pharmacogenomics: the genetics of variable drug responses. Circulation. 2011; 123:1661-70.

10. Mullard A. 2014 FDA drug approvals. Nat Rev Drug Discov. 2015;14:77-81.

11. Food and Drug Administration. FDA Clears Genetic Test that Advances Personalized Medicine Test Helps Determine Safety of Drug Therapy. FDA News. Rockville, MD: Food and Drug Administration; 2005. p. 5-53.

12. U.S. Food and Drug Administration. Table of Pharmacogenomic Biomarkers in Drug Labeling. Rockville, MD: U.S. Food and Drug Administration; 2019.

13. Haga SB, Burke W. Pharmacogenetic testing: not as simple as it seems. Genet Med. 2008;10:391-5

14. Scuteri A, Sanna S, Chen WM, Uda M, Albai G, Strait J, et al. Genome-wide association scan shows genetic variants in the FTO gene are associated with obesity-related traits. PLoS Genet. 2007;3:e115

15. Bush WS, Crosslin DR, Owusu-Obeng A, Wallace J, Almoguera $B$, Basford MA, et al. Genetic variation among 82 pharmacogenes: the PGRNseq data from the eMERGE network. Clin Pharmacol Ther. 2016;100:160-9.

16. Carpenter JS, Rosenman MB, Knisely MR, Decker BS, Levy KD, Flockhart DA. Pharmacogenomically actionable medications in a safety net health care system. SAGE Open Med. 2016; 4:2050312115624333.

17. Johnson JA, Klein TE, Relling MV. Clinical implementation of pharmacogenetics: more than one gene at a time. Clin Pharmacol Ther. 2013:93:384-5.

18. Whirl-Carrillo M, McDonagh EM, Hebert JM, Gong L, Sangkuhl $\mathrm{K}$, Thorn CF, et al. Pharmacogenomics knowledge for personalized medicine. Clin Pharmacol Ther. 2012;92:414-7.

19. Relling MV, Klein TE. CPIC: clinical pharmacogenetics implementation consortium of the pharmacogenomics research network. Clin Pharmacol Ther. 2011;89:464-7

20. Caudle KE, Hoffman JM, Whirl-Carrillo M, Haidar CE, Crews KR, Klein TE, et al. The clinical pharmacogenetics implementation consortium (CPIC): facilitating the adoption of pharmacogenetics into routine clinical practice and the electronic health record. Pharmacotherapy. 2014;34:E251-2.
21. Kimmel SE, French B, Kasner SE, Johnson JA, Anderson JL, Gage $\mathrm{BF}$, et al. A pharmacogenetic versus a clinical algorithm for warfarin dosing. N Engl J Med. 2013;369:2283-93.

22. Pirmohamed $M$, Burnside $G$, Eriksson $N$, Jorgensen $A L$, Toh $C H$ Nicholson T, et al. A randomized trial of genotype-guided dosing of warfarin. N Engl J Med. 2013;369:2294-303.

23. Johnson JA, Caudle KE, Gong L, Whirl-Carrillo M, Stein CM Scott SA, et al. Clinical pharmacogenetics implementation consortium (CPIC) guideline for pharmacogenetics-guided warfarin dosing: 2017 update. Clin Pharmacol Ther. 2017 102:397-404

24. Pratt V, McLeod H, Rubinstein W, Dean L, Kattman B, Malheiro A. Medical genetics summaries. Natl Cent Biotechnol Inf. 2018; 2:517-22.

25. Gaedigk A, Sangkuhl K, Whirl-Carrillo $M$, Klein T, Leeder JS. Prediction of CYP2D6 phenotype from genotype across world populations. Genet Med. 2017;19:69-76.

26. Clancy JP, Johnson SG, Yee SW, McDonagh EM, Caudle KE, Klein $T E$, et al. Clinical pharmacogenetics implementation consortium (CPIC) guidelines for ivacaftor therapy in the context of CFTR genotype. Clin Pharmacol Ther. 2014;95:592-7.

27. Scott SA, Sangkuhl K, Stein CM, Hulot JS, Mega JL, Roden DM, et al. Clinical pharmacogenetics implementation consortium guidelines for CYP2C19 genotype and clopidogrel therapy: 2013 update. Clin Pharmacol Ther. 2013;94:317-23.

28. Shah RR, Gaedigk A. Precision medicine: does ethnicity information complement genotype-based prescribing decisions? Ther Adv Drug Saf. 2018;9:45-62.

29. Suarez-Kurtz G, Parra EJ. Population diversity in pharmacogenetics: a Latin American perspective. Adv Pharmacol. 2018; 83:133-54

30. Villegas-Torres B, Sánchez-Girón F, Jaramillo-Villafuerte K, Soberón X, Gonzalez-Covarrubias V. Genotype frequencies of VKORC1 and CYP2C9 in native and Mestizo populations from Mexico, potential impact for coumarin dosing. Gene. 2015. 558:235-40

31. Gonzalez-Covarrubias V, Urena-Carrion J, Villegas-Torres B, Cossío-Aranda JE, Trevethan-Cravioto S, Izaguirre-Avila R, et al. Pharmacogenetic variation in over 100 genes in patients receiving acenocumarol. Front Pharmacol. 2017;8:863.

32. Roman YM. Race and precision medicine: is it time for an upgrade? Pharmacogenomics J. 2019;19:1-4.

33. Nebert DW, Menon AG. Pharmacogenomics, ethnicity, and susceptibility genes. Pharmacogenomics J. 2001;1:19-22

34. O'Donnell PH, Danahey K, Ratain MJ. The outlier in all of Us: why implementing pharmacogenomics could matter for everyone. Clin Pharmacol Ther. 2016;99:401-4.

35. Van Driest SL, Shi Y, Bowton EA, Schildcrout JS, Peterson JF, Pulley J, et al. Clinically actionable genotypes among 10,000 patients with preemptive pharmacogenomic testing. Clin Pharmacol Ther. 2014;95:423-31.

36. Jonsson A, Ladenvall C, Ahluwalia TS, Kravic J, Krus U, Taneera J, et al. Effects of common genetic variants associated with type 2 diabetes and glycemic traits on $\alpha$-and $\beta$-cell function and insulin action in humans. Diabetes. 2013;62:2978-83.

37. Martin AR, Gignoux CR, Walters RK, Wojcik GL, Neale BM, Gravel $\mathrm{S}$, et al. Human demographic history impacts genetic risk prediction across diverse populations. Am J Hum Genet. 2017; 100:635-49

38. Moreno-Estrada A, Gignoux CR, Fernández-López JC, Zakharia $\mathrm{F}$, Sikora M, Contreras AV, et al. Human genetics. The genetics of Mexico recapitulates native American substructure and affects biomedical traits. Science. 2014:344:1280-5

39. Contreras AV, Monge-Cazares T, Alfaro-Ruiz L, HernandezMorales S, Miranda-Ortiz H, Carrillo-Sanchez K, et al. Resequencing, haplotype construction and identification of novel variants of CYP2D6 in Mexican mestizos. Pharmacogenomics. 2011;12:745-56.

40. Bonifaz-Peña V, Contreras AV, Struchiner CJ, Roela RA, FuruyaMazzotti TK, Chammas R, et al. Exploring the distribution of genetic markers of pharmacogenomics relevance in Brazilian and Mexican populations. PLoS One. 2014;9:640-51.

41. Alcazar-González GA, Calderón-Garcidueñas AL, Garza-Rodríguez ML, Rubio-Hernández G, Escorza-Treviño S, Olano-Martin $\mathrm{E}$, et al. Comparative study of polymorphism frequencies of the CYP2D6, CYP3A5, CYP2C8 and IL-10 genes in Mexican and Spanish women with breast cancer. Pharmacogenomics. 2013; 14:1583-92

42. Maier L, Pruteanu M, Kuhn M, Zeller G, Telzerow A, Anderson $\mathrm{EE}$, et al. Extensive impact of drugs on human gut bacteria. Nature. 2018;555:623-8. 